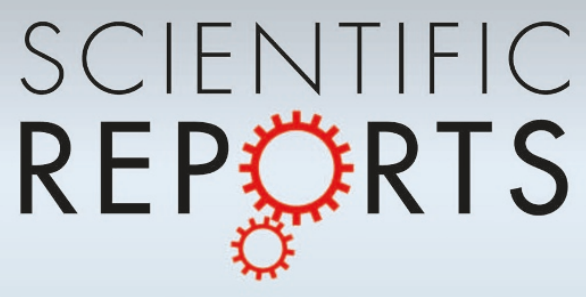

\title{
OPEN Advanced understanding of stickiness on superhydrophobic surfaces
}

SUBJECT AREAS:

FLUID DYNAMICS

MECHANICAL ENGINEERING

MECHANICAL PROPERTIES

NANOFLUIDICS

Received

11 July 2013

Accepted

29 October 2013

Published

20 November 2013

Correspondence and requests for materials should be addressed to

J.W. (lancejunwu@ gmail.com) or J.X.

(xiajun@seu.edu.cn)

\author{
Jun Wu, Jun Xia, Wei Lei \& Bao-ping Wang
}

School of Electronic Science and Engineering, Southeast University, China, 210096.

This study explores how contact angle hysteresis and titling angle relate with stickiness on superhydrophobic surfaces. The result indicates that contact angle hysteresis could not be mentioned as a proper factor to evaluate the surface stickiness. By analyzing the system pinning force of droplet placed on a titled surface, we concluded that both solid fraction and surface geometric factor are the critical factors determining the surface stickiness.

○

ver last decades, some special surfaces in nature feature high water contact angle $\left(>150^{\circ}\right)$ attracted enormous interests for both fundamental research and practical application ${ }^{1-8}$. Some of these surfaces, lotus leaves for instance, not only exhibit high water contact angle, but also have extremely low sliding angle, which cause water droplets to bead and roll off from the surface by slightly titling it ${ }^{1}$. This behavior provides extreme water repellency and self-cleaning characteristics ${ }^{2-5}$. Meanwhile, some other surfaces, i.e. rose-petal surface, with large water droplet sliding angles have the ability to make water droplet pinned on the surfaces at any tilting angle (TA $)^{6}$. Such high-adhesion surfaces also possess many potential applications, no-loss fluid transportation for example ${ }^{7,8}$. According to the different values of TA, the above mentioned surfaces fall into two categories, which are "slippery" and "sticky" ones, featuring low TA and high TA respectively, all these surfaces are uniformly named as "superhydrophobic" surfaces in litheture though.

Meanwhile, people found that there exists a wide range of "metastable" contact angles when a liquid meniscus scans the solid surface? . Because there are free energy barriers which exist between these metastable states, a true "equilibrium" contact angle is almost impossible to measure in real time. The famous Wenzel's and Cassie's theories are therefore only valuable in predicting the thermodynamically stable contact angles in theory ${ }^{10,11}$. Therefore, contact angle hysteresis ( $\mathrm{CAH})$ defined as the difference of advancing angle and receding angle is usually measured to fully characterize any surface ${ }^{12}$. Recently, however, it seems CAH has a new function and already somehow been treated as a criterion to evaluate the stickiness of superhydrophobic surfaces by researchers and students ${ }^{13-16}$. People believe that increase in stickiness will absolutely result in a corresponding increase in $\mathrm{CAH}$. As mentioned in the previous section, TA value has widely been used to describe the surface stickiness. Since both these two parameters have been mentioned in publications to describe the same physical phenomena, it is also naturally concluded by researchers that TA and CAH share the same changing tendency with the stickiness ${ }^{15}$. However, we achieved an opposite conclusion according to our recent experimental investigation. We fabricated several posts arrays with different solid fractions. The TA increases with the solid fraction, which proves that the stickiness/pinning force increases with solid fraction ${ }^{17-19}$. Nevertheless, the CAH shows a decreasing tendency while the solid fraction increases. This result is of particular interest from theory point of view. It reveals that $\mathrm{CAH}$ may not be the proper factor to describe the surface stickiness, and it is not necessary to take extra effects to make low CAH surface to achieve good slip performance.

\section{Results}

Figure 1 shows the post patterns fabricated by us. To quantitatively identify the effect of solid fraction on stickiness, we also calculated the solid fraction of the four samples, which vary from (a) 0.06 , (b) 0.18 , and (c) 0.25 to (d) 0.50 . The calculation method is indicated in Figure 2 (a). From the observation of apparent contact angles measurements by the sessile drop method (Method Section for details), the droplet boundary is pinned to the substrate in the initial stage. Therefore, the contact radius of the droplet remains constant while the contact angle gradually increases or decreases. Such a pinning mode then transits to a depinning mode with an increase or decrease of contact radius, as illustrated in Figure 2 (b) and (c). During this process, both advancing angle $\theta_{\mathrm{a}}$ and receding angle $\theta_{\mathrm{r}}$, and therefore $\mathrm{CAH}$ were determined. Please note that the volume of water droplet adopted in 


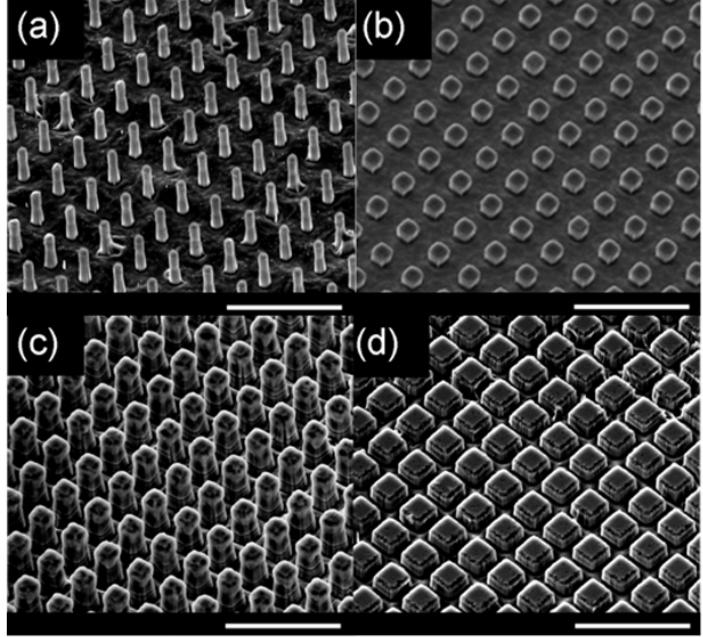

Figure 1 Scanning electron microscopy images of the square posts. The liquid-solid contact area fraction varies from (a) 0.06 ; (b) 0.18 ; (c) 0.25 ; (d) 0.50 . The scale bars are $20 \mu \mathrm{m}$.

our experiments is 1 microlitre. This value is selected to provide relatively reliable results by eliminating the influences of gravity effect on "bulky" droplets profile and the evaporation speed on "tiny" droplets.

According to the thermodynamical analysis, the triple-phase contact line (TCL) of water droplet would "select" one certain location to minimize the system energy. Meanwhile, this TCL location determines a theoretically stable contact angle, which is predicted by Cassie theory. The difference between this Cassie angle and advancing/receding angles indicate that there exists pinning force which prevents TCL moving smoothly to that specific location. Droplet pinning lasts until the apparent contact angle reaches to the advancing or receding value. The unbalanced surface tension due to the difference between the Cassie angle and advancing/receding angle results in the driving force for depinning motion of the droplet boundary, and the depinning force per unit length of the apparent droplet boundary can be expressed as ${ }^{20}$

$$
F_{P}=\gamma\left|\cos \theta_{X}-\cos \theta_{C}\right|
$$

Where $\gamma$ is the surface tension of liquid $(\gamma=72 \mathrm{mN} / \mathrm{m}$ for water at $23^{\circ} \mathrm{C}$ ), $\theta_{X}$ could be advancing angle $\theta_{a}$ or receding angle $\theta_{r}$ depends on situation.

Figure 3(a) shows the CAH values on each surface, and the pinning forces calculated according to equation (1), with respect to the liquid-solid contact area fraction. According to the "common sense", it is expected that the surface with smaller solid fraction should have smaller pinning force, and therefore smaller CAH. Although there is no convincing theory explaining this logic, it has been widely accepted by researchers. Therefore, researchers kept claiming that they achieved "slippery surface with extremely low CAH"13-16. In our case, the pinning force does decline dramatically with the decrease of the solid contact area fraction as indicated in Figure 3(a). However, the result presented herein indicates that the $\mathrm{CAH}$ values increase while the solid fraction decreases, which seems unreasonable as it is contrary to the "common sense". But interestingly, this "common sense" is not challenged for the first time, Zhang et al even found that the $\mathrm{CAH}$ is not dependent on the solid fraction, because they observed different $\mathrm{CAH}$ values from two different patterns with the same solid fraction ${ }^{17}$. Both these two results imply that $\mathrm{CAH}$ could not be simply used to evaluate the surface stickiness.

\section{Discussion}

To well explain the previously mentioned results and get an advanced understanding of stickiness on superhydrophobic surfaces, we need to address one question first: how the surface with larger $\mathrm{CAH}$ has smaller pinning force? Actually, according to equation (1), the pinning force is determined by the difference of cosine values other than $\mathrm{CAH}$. The cosine values decrease with the increasing contact angles. The contact angles on our samples are in the range from $90^{\circ}$ to $180^{\circ}$. For a certain CAH value in this range, the difference of cosine values

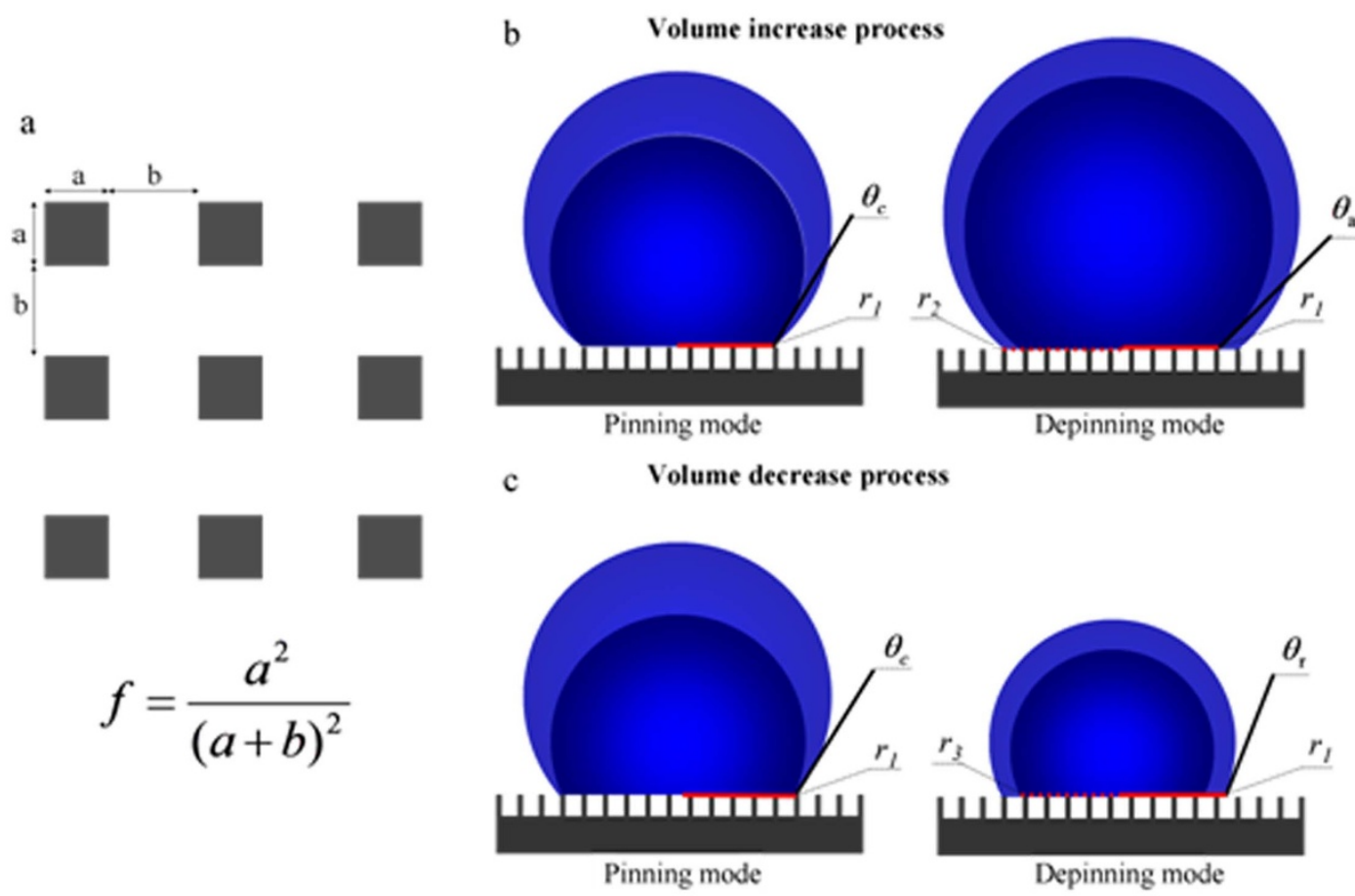

Figure $2 \mid$ (a) The calculation method of solid fraction, and the pinning and depinning phenomena of (b) a droplet with increasing volume and (c) a droplet with decreasing volume. A pinning mode transits to a depinning mode during the volume increase or decrease process, when the apparent contact angle reaches to advancing angle $\theta_{\mathrm{a}}$ or receding angle $\theta_{r}$. 

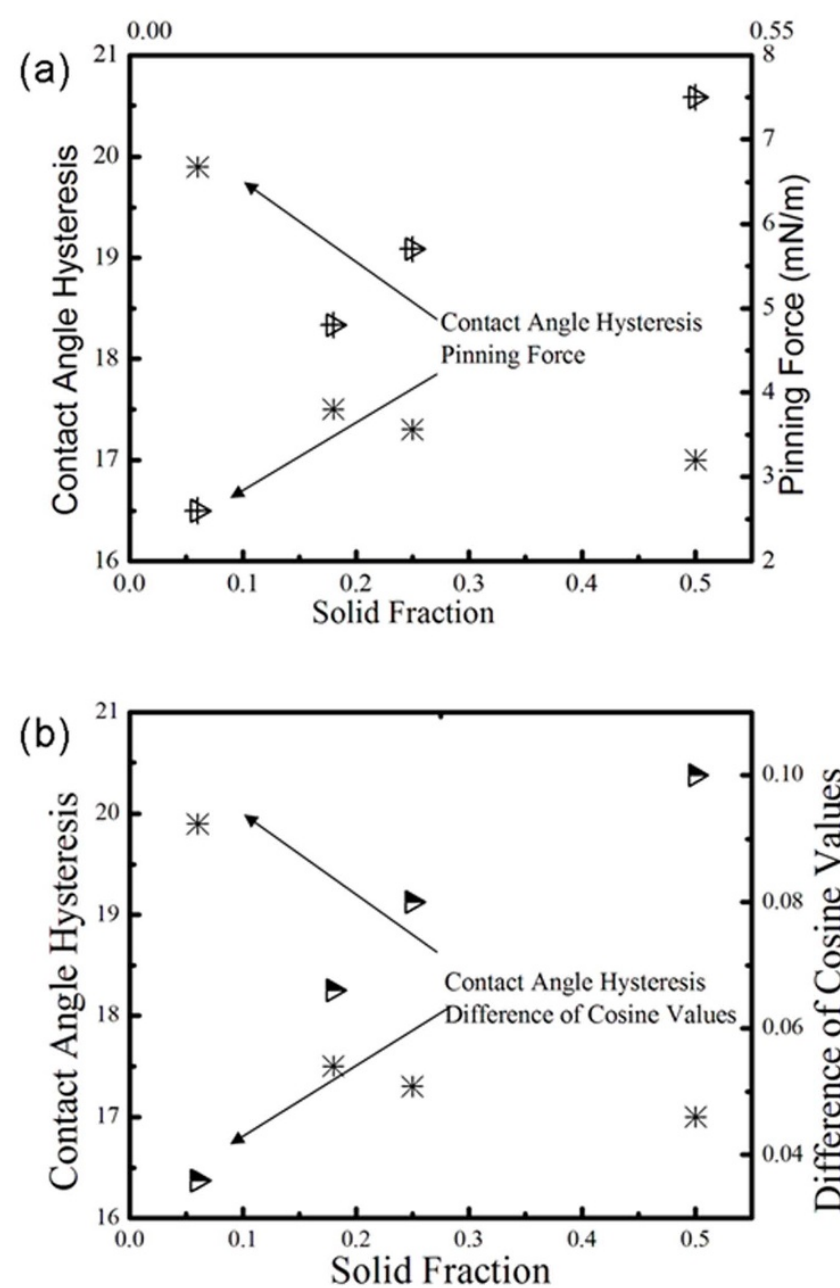

Figure 3 | (a) Contact angle hysteresis and pinning force (b) contact angle hysteresis and the difference of cosine values on the pattern superhydrophobic surfaces. Both pinning forces and the difference of cosine values have opposite changing tendency with contact angle hysteresis.

and the corresponding pinning force will decrease with the increasing contact angles. Furthermore, although the measured CAH values increase with the decreasing solid fractions, the difference of cosine values on the smallest solid fraction point is still much lower than others. Actually, as can be observed in figure 3(b), difference of cosine values at the smallest solid fraction point is only $\sim 1 / 30$ of the one at the largest solid fraction point. Therefore, it is reasonable to observe the coexistence of lower pinning force and larger $\mathrm{CAH}$ in Figure 3 (a)

In the following section, we would like to discuss more on this topic in this communication. The above mentioned pinning force is just the one applied on per unit length of the apparent droplet boundary. To evaluate the surface stickiness precisely, it is critical to measure the total pinning force applied on the whole system. According to previous studies ${ }^{23}$, the system pinning force can be expressed by the relation:

$$
F_{S P}=\operatorname{mg} \sin \theta_{T}
$$

Where $\mathrm{m}$ is the mass of the liquid droplet, $\mathrm{g}$ is gravitational acceleration, $\theta_{T}$ represents TA.

Figure 4 (a) shows the TA values on each surface, together with the system pinning force, with respect to the liquid-solid contact area fraction. For all the samples, the TA simply demonstrates the same changing tendency with system pinning force.

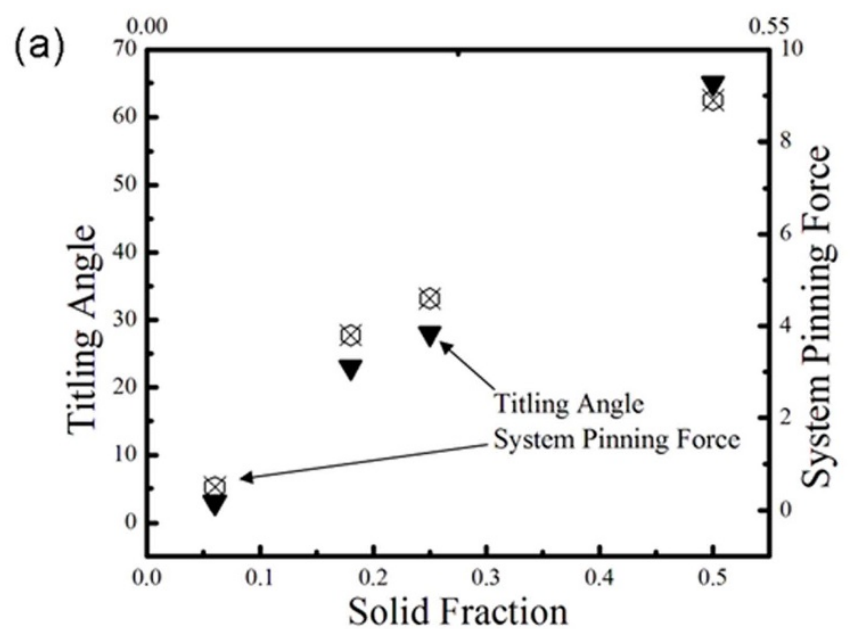

(b)

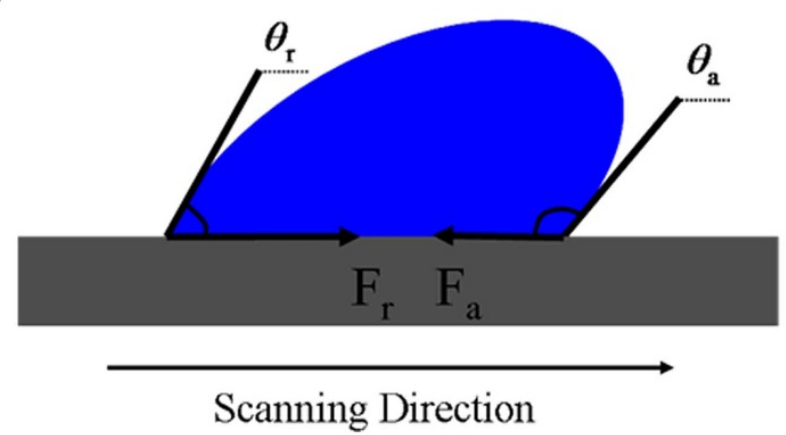

Figure $4 \mid$ (a) The titling angles and system pinning force on the pattern superhydrophobic surfaces, and (b) the sketch of scanning droplet on solid surface.

McCarthy et al have commented on the importance of the TCL and its pinning effect on surface stickiness ${ }^{21}$. Meanwhile, they challenged the reasonableness of adopting solid fraction as the critical influence factor on the stickiness ${ }^{22}$. However, for their own theory, there is no direct correlation between the surface structures and surface stickiness could be revealed. To explore the working mechanism and also the critical influence factors of this relation, we further considered the system pinning force when droplet scanning over a solid surface. As indicated in Figure 4 (b), the droplet will not scan until the driving force by surface tension forces is large enough to challenge this pinning force, therefore this system pinning force can also be expressed as:

$$
F_{S P}=F_{\mathrm{r}}-F_{a}=L \gamma_{L}\left(\cos \theta_{r}-\cos \theta_{a}\right)
$$

By combining Eq. (2) and Eq. (3), we could build a new relation between titling angle and TCL:

$$
\operatorname{mg} \sin \theta_{T}=L \gamma_{L}\left(\cos \theta_{r}-\cos \theta_{a}\right)
$$

Note that $\mathrm{L}$ could not be simply treated as the width of the drop viewed along the sliding direction. $\mathrm{L}$ is a geometric factor depends on the pattern types, determines the effective TCL length along the droplet boundary, on which the surface tension forces are effectively applied. Based on this relation, the observed increase in TA with increasing solid fraction arises from two main factors. Firstly, the droplet contact angle decreases with increasing solid fraction, which would cause the footprint area of droplet to increase. At the same time, the parameter L in Eq.(4) is increased. Secondly, as discussed in previous section, the difference of cosine values increases while the contact angle decreases (Figure 3 (b)). Therefore, the parameter of solid fraction not only affected the effective TCL length $\mathrm{L}$ along the 
droplet boundary, but also influenced the pinning effect on per unit length of the apparent droplet boundary. Meanwhile, however, as mentioned in previous section, neither the effective TCL length L nor the pinning effect on per unit length of the apparent droplet boundary could be totally determined by solid fraction, they are also closely related with the surface pattern types. We believe that different surface geometries will definitely induce totally different pinning effects, even though these surfaces have the same solid fraction. Our point is well in line with the experimental results of Zhang ${ }^{17}$. All these results and discussion indicate that the surface stickiness should be determined by overall considering the solid fraction and also the surface geometric factor. The more detailed working mechanism determining the value of $\mathrm{L}$ should be of interests to those researchers in this field and be investigated further.

By investigating the relation between $\mathrm{CAH}$, TA and pinning force, this study reveals the proper influence factors and the related working mechanism of the pinning phenomena on superhydrophobic surfaces. The pinning force which prevents TCL moving smoothly and forming the thermodynamically stable contact angle is found not directly related with $\mathrm{CAH}$. However, we found the surface stickiness depends on the morphology of the surface structures, which includes the parameters of solid fraction and surface geometric factor. This new insight paves the way to better understand the outstanding problem of hysteresis, pinning. Not only for the theory development, is the surface stickiness also of significant importance in the microfluidics applications, such as friction reduction.

\section{Methods}

Surfaces structures consisting of square posts with the same period of $7 \mu \mathrm{m}$ were fabricated on silicon wafers. We first make a template of the structure on a photoresist film coated on Si by photolithography. The template in the photoresist was then transferred to the Si underneath by Gold-Assistant-Etching. Our studies used square structures with width of $1.7,3,3.5$ and $5 \mu \mathrm{m}$. We characterized the three-dimensional structure of the textures by scanning electron microscopy (SEM). To make the surface hydrophobic, we silanized them by perfluorooctyl trichlorosilane (C8H4Cl3F13Si) vapor. In brief, we put the silicon substrates into a PECVD to remove the organic contaminants by $\mathrm{O} 2$ plasma. Then the substrate was put into a chamber, in which a small glass beaker containing liquid $\mathrm{C} 8 \mathrm{H} 4 \mathrm{Cl} 3 \mathrm{~F} 13 \mathrm{Si}$ was placed. Thereafter the chamber was vacuum pumped to about $10^{-1}$ torr overnight to allow the trichlorosilane group of the $\mathrm{C} 8 \mathrm{H} 4 \mathrm{Cl} 3 \mathrm{~F} 13 \mathrm{Si}$ molecules to react with the silanol group of the silicon. In this study, substrates were used within two weeks after silanization. The apparent contact angles of water droplet on these patterns were determined by the sessile drop method with a contact angle meter. In detail, water droplets of $1 \mu \mathrm{L}$ were firstly placed onto the samples gently at a room condition, the volume of water droplets is then increased or decreased continually and slowly through a needle inserted from top center, meanwhile, the droplet profiles are observed and recorded to provide the information of contact angle and contact radius of the droplets. In another case, TA on these samples was also measured. The sample substrate was firstly mounted onto a rotatable operating stage. Then a $1 \mu \mathrm{L}$ water droplet was dispersed onto the substrate whereupon the stage was rotated slowly. The angle at which the droplet began to slide down was noted as the TA.

1. Johnson, R. E. \& Dettre, R. H. Contact angle hysteresis. 3. study of an idealized heterogeneous surface. J. Phys. Chem. 68, 1744-1750 (1964).

2. Wu, J. et al. A hierarchical mesh film with superhydrophobic and superoleophilic properties for oil and water separation. J. Chem. Technol. Biotechnol. 87, 427-430 (2012).

3. Feng, X. J. \& Jiang, L. Design and Creation of Superwetting/Antiwetting Surfaces. Adv. Mater. 18, 3063-3078 (2006).

4. Xin, B. W. \& Hao, J. C. Superhydrophobic self-assembled monolayers of longchain fluorinated imidazolium ionic liquids. RSC. Advances. 2, 5141-5146 (2012).

5. Wu, J., Xia, J., Lei, W. \& Wang, B. P. Superhydrophobic surface based on a corallike hierarchical structure of ZnO. PLoS. One. 5, e14475 (2010).
6. Wang, J. M., Yang, Q. L., Wang, M. C., Wang, C. \& Jiang, L. Rose petals with a novel and steady air bubble pinning effect in aqueous media. Soft. Matter. 8, 2261-2266 (2012).

7. Hong, X., Gao, X. F. \& Jiang, L. Application of superhydrophobic surface with high adhesive force in no lost transport of superparamagnetic microdroplet. J. Am. Chem. Soc. 129, 1478 (2007).

8. Cho, W. K. \& Choi, I. S. Fabrication of Hairy Polymeric Films Inspired by Geckos: Wetting and High Adhesion Properties. Adv. Funct. Mater. 18, 1089-1096 (2008).

9. Zhang, X. Y. \& Mi, Y. L. Dynamics of a Stick-Jump Contact Line of Water Drops on a Strip Surface. Langmuir. 25, 3212-3218 (2009).

10. Cassie, A. B. D. \& Baxter, S. Wettability of porous surfaces. Trans. Faraday. Soc. 40, 546-551 (1944).

11. Wenzel, R. N. Resistance of solid surfaces to wetting by water. Ind. Eng. Chem. Res. 28, 988-994 (1936)

12. Yarnold, G. D. The hysteresis of the angle of contact of mercury. Proc. Phys. Soc Lon. 58, 120-127 (1946).

13. Konrad, W., Ebner, M., Traiser, C. \& Roth-Nebelsick, A. Leaf surface wettability and implications for drop shedding and evaporation from forest canopies. Pure. Appl. Geophys. 169, 835-845 (2012).

14. Liu, Y. \& Choi, C. H. Condensation-Induced Wetting State and Contact Angle Hysteresis on Superhydrophobic Lotus Leaves. Colloid. Polym. Sci. 291, 437-445 (2013).

15. Wolfs, M., Darmanin, T. \& Guittard, F. Analogy of morphology in electrodeposited hydrocarbon and fluorocarbon polymers. RSC. Advances. 3, 647-652 (2013).

16. Dufour, R. et al. Engineering sticky superomniphobic surfaces on transparent and flexible PDMS substrate. Langmuir. 26, 17242-17247 (2010).

17. Zhang, X. Y. et al. Effect of pattern topology on the self-cleaning properties of textured surfaces. J. Chem. Phys. 127, 014703 (2007).

18. Wu, J., Xia, J., Zhang, Y. N., Lei, W. \& Wang, B. P. A simple method to fabricate the different extents of superhydrophobic surfaces. Physica E. 42, 1325-1328 (2010).

19. Xu, W. \& Choi, C. H. From sticky to slippery droplets: dynamics of contact line depinning on superhydrophobic surfaces. Phys. Rev. Lett. 109, 024504 (2012).

20. Sefiane, K. Effect of nonionic surfactant on wetting behavior of an evaporating drop under a reduced pressure environment. J. Colloid. Interface. Sci. 272, 411-419 (2004).

21. Wier, K. A. \& McCarthy, T. J. Condensation on Ultrahydrophobic Surfaces and Its Effect on Droplet Mobility: Ultrahydrophobic Surfaces Are Not Always Water Repellant. Langmuir. 22, 2433-2436 (2006).

22. Gao, L. C. \& McCarthy, T. J. How Wenzel and Cassie were wrong. Langmuir. 23, 3762-3765 (2007).

23. Brown, R. A., Orr, F. M. \& Scriven, L. E. Static drop on an inclined plate-analysis by the finite-element method. J. Colloid. Interface. Sci. 73, 76-87 (1980).

\section{Acknowledgments}

This research was supported by the National Basic Research Program of China (2013CB328803; 2010CB327705); the National High Technology Research and Development Program of China (2012AA03A302; 2013AA011004); National Natural Science Foundation of China $(61306140,51202028)$; China Postdoctoral Science Foundation (2013M530222); Natural Science Foundation of Jiangsu Province (BK20130618); and Jiangsu Planned Projects for Postdoctoral Research Funds (1301097C).

\section{Author contributions}

J.W. conceived and designed the research. J.X. supervised the project. J.W. carried out theoretical calculations and experiments. J.W. wrote the manuscript. W.L. and B.P.W. participated in the discussions and reviewed the manuscript. All authors analyzed the data and reviewed the manuscript.

\section{Additional information}

Competing financial interests: The authors declare no competing financial interests.

How to cite this article: Wu, J., Xia, J., Lei, W. \& Wang, B.-P. Advanced understanding of stickiness on superhydrophobic surfaces. Sci. Rep. 3, 3268; DOI:10.1038/srep03268 (2013).

This work is licensed under a Creative Commons Attribution-

NonCommercial-NoDerivs 3.0 Unported license. To view a copy of this license, visit http://creativecommons.org/licenses/by-nc-nd/3.0 